\title{
Moderate Cardiac Valve Stenosis
}

National Cancer Institute

\section{Source}

National Cancer Institute. Moderate Cardiac Valve Stenosis. NCI Thesaurus. Code C99990.

The cardiac valve orifice is abnormally narrow, to a moderate degree. (ACC) 